\title{
Increasing the Throughput of Wireless Mesh Networks with Cooperative Techniques
}

\author{
Christian Ibars* Aitor del Coso* \\ *Centre Tecnològic de Telecomunicacions \\ de Catalunya - CTTC \\ Parc Mediterrani de la Tecnologia \\ Av. del Canal Olímpic s/n \\ 08860 Castelldefels - Spain
}

\author{
Yan Grunenberger $^{\dagger}$ Fabrice Theoleyre ${ }^{\dagger}$ Franck Rousseau $^{\dagger}$ \\ ${ }^{\dagger}$ Laboratoire Informatique de Grenoble - LIG \& Institut National \\ Polytechnique de Grenoble - INPG \\ 681 rue de la Passerelle \\ BP 72
}

38402 Saint Martin d'Hres Cedex, France

\begin{abstract}
Wireless mesh networks can increase wireless connectivity by routing packets through multiple wireless hops. However, a very efficient wireless interconnection is necessary to make these networks operative in practice. In this paper we describe a technique to increase the throughput of wireless mesh networks, based on cooperative communications. In the proposed setup, multiple nodes cooperate at the physical layer by jointly transmitting or receiving data. In particular, two cooperative strategies are proposed: opportunistic relaying, and partial decoding. In order to integrate such transmission scheme in a mesh network, each cooperative set of nodes is organized in a unit called virtual link. Virtual links can be seen by the network layer as regular links, which hides the cooperative strategy from the routing algorithm. Simulation results for Rayleigh and Rice fading show remarkable gains of the cooperative strategies with respect to non-cooperative transmission.
\end{abstract}

\section{INTRODUCTION}

Wireless networks have been traditionally designed as access networks to a wired communications network such as PSTNs or the Internet. However, such systems fail to provide sufficient connectivity and coverage, and require huge investments for the wired infrastructure. Recently, there has been a growing interest in extending wireless connectivity. Practical examples are community networks [1] or, more generally, wireless mesh networks. In such networks, the efficient design of wireless multi-hop transmission is very important. It has been recognized in the literature that an effort to optimize multi-hop transmission must be done on several layers [2], [3], [4] of the OSI stack.

At the same time, from the physical layer perspective, there has also been growing interest in studying multi-terminal communications; initial results derived in [5] for the relay channel, which involves a source, a destination, and an intermediate node, were revisited and extended to multiple relays [6] and to multiple, cooperating sources [7]. Since then, power and resource allocation schemes, as well as suitable encoding and signal processing schemes, have been proposed in the literature. All these schemes are referred to in general as cooperative communications techniques.

In this paper we propose a method to integrate cooperative techniques in wireless mesh networks in order to increase their throughput. We assume that the network is subdivided into an access subnetwork and a high-speed backhaul interconnection. For the backhaul interconnection, we propose to substitute the current concept of a link between two network nodes by a more general concept of virtual link, which may involve several nodes that coordinate to communicate cooperatively. For each virtual link, we propose an optimal use of spectrum and power under two different techniques: opportunistic relaying and partial decoding. A linear network formed by several virtual links is evaluated in terms of end-to-end average throughput and, for delay-sensitive applications, in terms of end-to-end outage probability. Simulation results show that remarkable gains are possible using cooperative techniques for multi-hop links.

The proposed system model is described in the next section. Section III describes the proposed cooperative backhaul. Section IV describes the proposed cooperative techniques, which optimally allocate spectrum usage and power to all transmitting nodes. Section V shows numerical results for the proposed scheme, and compares it to non-cooperative multihop transmission. Finally, Section VI contains the conclusions.

\section{SySTEM MODEL}

We envision a wireless mesh network composed of heterogeneous nodes, ranging from energy-constrained mobile devices to high performance fixed nodes. In order to meet reasonable delay and throughput requirements, such a network relies on more capable nodes to route high volumes of traffic in what we define as a wireless backhaul interconnection. Each node in the wireless backhaul is also an access point, which acts as gateway of its access sub-network. Some of the wireless backhaul nodes are gateways to the wired Internet. In this paper we study a simple, linear configuration for the wireless backhaul such as the one shown in Figure 1 (a ring topology could be studied similarly).

In our setup, we assume that node 1 wants to send information to node $N$ (gateway node) through the wireless backhaul. In general, these two nodes may not communicate directly. Let $d$ denote the distance between two consecutive backhaul nodes, $\alpha$ the path loss exponent, and $P$ the transmitted power. When node $i$ transmits, the received power at node $j>i$ is 


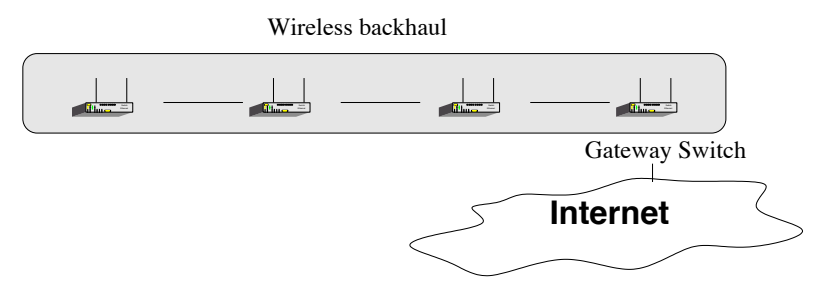

Fig. 1. Linear wireless backhaul topology.

given by

$$
P_{r}=h((j-i) d)^{-\alpha} P
$$

where $h$ denotes a random channel gain with unit variance, which is distributed according to a Rayleigh or Rice amplitude distribution, depending on whether the nodes are in line-ofsight or not. With this channel model, we define the carrier sensing $(C S)$ region around node $i$ as a circle with radius

$$
r_{C S}=\left(\frac{P}{\gamma_{C S}}\right)^{\frac{1}{\alpha}}
$$

which determines the distance at which the average received power equals a predefined threshold $\gamma$. All other nodes detecting an active transmission with average power $P_{r}>\gamma_{C S}$ do not transmit in order to avoid interfering node $i$. The number of nodes inside the CS region determines the schedule of a multihop transmission along the network. Similarly, we define the Connectivity region as

$$
r_{C o n}=\left(\frac{P}{\gamma_{C o n}}\right)^{\frac{1}{\alpha}}
$$

where $\gamma_{C o n}$ is the minimum received power that allows two nodes to communicate (at the lowest data rate). According to the model, $r_{C S}$ and $r_{C o n}$ are determined by the distance between nodes, the transmitted power, and $r_{C o n}$ also by the data rate. In our model, we assume that both $r_{C S}$ and $r_{C o n}$ fall within $2 d$ and $3 d$, so that, when node $i$ is transmitting, nodes $i+1$ and $i+2$ fall within $r_{C S}$ and $r_{C o n}$.

\section{Cooperative Wireless Backhaul}

In order to define the proposed cooperative wireless backhaul, we assume that a conventional routing algorithm, which minimizes the number of hops, is used. Since nodes $i+1$ and $i+2$ are reachable from node $i$ according to the system model, the transmission on each hop will skip one intermediate node. Although intermediate nodes do not participate in the communication, they may not transmit nor receive since they fall within the CS region of the transmitting node. We propose to establish a cooperative strategy between transmitting, receiving, and intermediate nodes, which we define as a virtual link. As in a conventional link, the virtual link is identified by a source node and a destination node. A local coordination mechanism can be put in place to use the intermediate node in a cooperative communication scheme. As shown in Figure 2, under such mechanism, the time slot allocated for the transmission of information is partitioned in two sub-slots: the

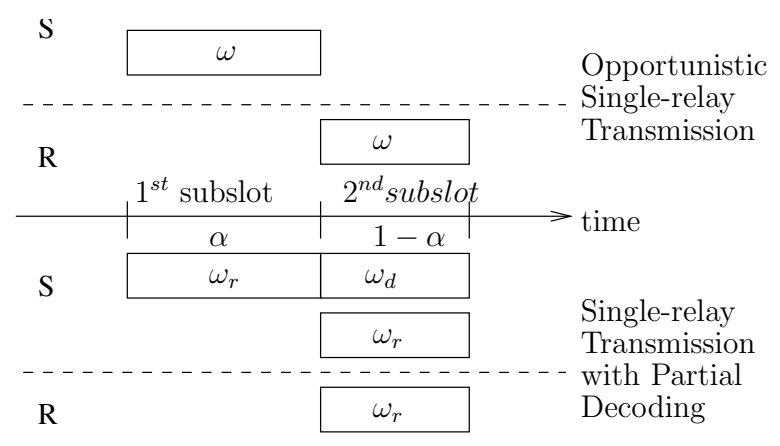

Fig. 2. Use of time slots on a virtual link.

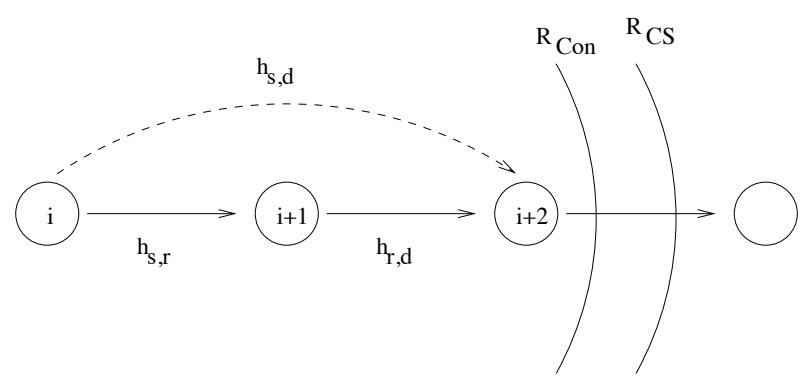

Fig. 3. Illustration of the virtual link concept.

intermediate node first listens to the transmission of the source node and then it retransmits it to the destination. Two different retransmission strategies are presented later in section IV.

The transmission proceeds in two steps: during the first step, only the source is transmitting while both the relay and the source can transmit data during the second step. However, the synchronization is implicitly set up by the source. We envision this complex transmission as a single MAC action. Thus, this scheme can be adapted to various access methods: random access, like CSMA, or deterministic fixed TDMA approaches. Once channel access is gained by a station, depending on the conditions and other higher level strategies, relaying can be used or not.

In order for the virtual link to be transparent to the rest of the network, the CS region must not change with respect to its corresponding conventional link. The idea of virtual link is illustrated in Figure 3. In the figure, the dashed line represents the link chosen by the routing algorithm, and solid lines show connections through intermediate links which may be used by the cooperative algorithms.

The operation of the cooperative wireless backhaul is as follows: nodes establish their list of neighbors based on their individual connectivity regions. For each link to a neighbor, a node tries to establish a virtual link with one or more neighbors, while satisfying the CS region for that link. If an appropriate cooperative setup for a virtual link is found, such setup is used whenever a transmission though that link is required. Going back to our linear model, a routing algorithm selecting the minimum number of hops will select a path going through every other link, which is the farthest within the 
connectivity region. Then, if appropriate, intermediate nodes will participate in the communication by taking part in a virtual link, according to one of the cooperative strategies described in the next section. It is important to note that, while this approach may be suboptimal, it hides the complexity of node cooperation from the network layer, since routing may be performed over actual links or virtual links indistinctively.

In order to evaluate the performance of the cooperative wireless backhaul with respect to a non-cooperative one, we define the following metrics:

- Throughput, under the assumption that nodes have a buffer with infinite size to store data:

$$
T_{\infty}=\min _{i}\left(E\left(R_{i}\right)\right)
$$

where $i=1,2, \cdots N, E()$ is the expectation operator, and the expectation is taken over the channel fading distribution; $R_{i}$ is the instantaneous data rate for link $i$. In this case, the throughput is limited by the minimum average rate of any link in the end-to-end path.

- Throughput, under the assumption that nodes may not store data and have to forward it inmediately

$$
T_{0}=E\left(\min _{i} R_{i}\right)
$$

Since nodes may not store data packets, the throughput is limited by the instantaneous rate of each link, which is the bottleneck of the end-to-end path; the average throughput is then obtained as its expectation.

- End-to-end outage probability (for a fixed rate $R$ ) as

$$
P_{o}=1-\prod_{i}\left(1-\operatorname{Pr}\left(R_{i}<R\right)\right)
$$

This metric is used to evaluate the performance of the network for constant bit rate traffic. For a given data rate $R$, the outage probability determines the probability that the end-to-end path is not available. It is obtained from the link outage probability, which depends on the instantaneous data rate $R_{i}$ allowed by the channel state.

In the following, throughput and data rates shall be obtained from the Shannon capacity of wireless links, and measured in bits per second per Hertz $(\mathrm{b} / \mathrm{s} / \mathrm{Hz})$. Notice that such measure is normalized by the system bandwidth; unnormalized throughput may be obtained multiplying the normalized value by the system bandwidth in $\mathrm{Hz}$.

We note that these throughput metrics do not take into account the scheduling of transmissions among consecutive nodes. When taking that into account, the throughput must be divided by a factor of 2, to account for half-duplex operation, if directional antennas are used. It must be divided by a higher factor otherwise, since backward nodes $i-1$ and $i-2$ also fall within the CS region of node $i$. Finally, a further throughput loss may be incurred by channel access protocols. In any case, such factor shall be equal for non-cooperative and cooperative strategies, and has no effect in the comparison of their performance. This issue will be discussed in more detail in the final paper.

\section{RELAYING STRATEGIES}

Two cooperative schemes are proposed for the virtual link: opportunistic single-relay transmission, and single-relay transmission with partial decoding [8]. Both are based on a halfduplex scheme where the relay node receives during one time slot, and transmits during the other.

\section{A. Opportunistic Single-Relay Transmission}

With opportunistic relay transmission, the source node transmits the data packet to the relay utilizing a first time slot of duration $\alpha \in[0,1]$. Due to the broadcast nature of the wireless medium, the signal also arrives to destination, which makes use of it when decoding at time slot 2 . In terms of Shannon capacity, the relay node is able to decode the packet if the transmission rate $R$ satisfies:

$$
\mathrm{R} \leq \alpha \cdot \log _{2}\left(1+\left|h_{s, r}\right|^{2} \frac{\mathrm{P}_{s}}{N_{o}}\right)
$$

where $h_{s, r}$ denotes the channel from the source to the relay and $\mathrm{P}_{s}$ is the power transmitted by the source. Then, during time slot 2, an opportunistic communication takes place: if the relay-destination channel is better than the source-destination channel, then the relay retransmits the data using an independently generated codeword of duration $1-\alpha$, while the source remains silent. Otherwise, the source retransmits the packet during time slot 2 and the relay does not participate. Using this scheme it is possible to obtain spatial diversity. Furthermore, the relay only participates in the transmission when it is beneficial. Finally, in order to decode, the destination node uses its received signals during both slots. Therefore, it can decode the packet if:

$$
\begin{aligned}
\mathrm{R} \leq & \alpha \cdot \log _{2}\left(1+\left|h_{s, d}\right|^{2} \frac{\mathrm{P}_{s}}{N_{o}}\right) \\
& +(1-\alpha) \log _{2}\left(1+\frac{\max \left\{\left|h_{s, d}\right|^{2} \mathrm{P}_{s},\left|h_{r, d}\right|^{2} \mathrm{P}_{r}\right\}}{N_{o}}\right)
\end{aligned}
$$

where $h_{s, d}$ and $h_{r, d}$ denote the channel from the source to the destination and from the relay to the destination, respectively, and $\mathrm{P}_{r}$ the power transmitted by the relay. Hence, aiming at jointly satisfying (7) and (8), the maximum transmission rate of the virtual link remains

$$
\begin{gathered}
\mathrm{R}=\max _{\alpha} \min \left\{\alpha \cdot \log _{2}\left(1+\left|h_{s, r}\right|^{2} \frac{\mathrm{P}_{s}}{N_{o}}\right),\right. \\
\alpha \cdot \log _{2}\left(1+\left|h_{s, d}\right|^{2} \frac{\mathrm{P}_{s}}{N_{o}}\right) \\
\left.+(1-\alpha) \log _{2}\left(1+\frac{\max \left\{\left|h_{s, d}\right|^{2} \mathrm{P}_{s},\left|h_{r, d}\right|^{2} \mathrm{P}_{r}\right\}}{N_{o}}\right)\right\}
\end{gathered}
$$

It is important to point out that the total transmitted power must be normalized in order that the CS region does not change when the relay is active. Therefore, at any distance $d_{l} \geq r_{C S}$ from the source, the power allocation for cooperative transmission must satisfy

$$
\mathrm{P}_{s} d_{l}^{-\alpha}+\mathrm{P}_{r}\left(d_{l}-d\right)^{-\alpha}=P
$$


where $P$ stands for the received power when non-cooperative transmission is used.

\section{B. Single-Relay Transmission with Partial Decoding}

With partial decoding, the source splits its data packet (of rate $\mathrm{R}$ ) in two data packets $\omega_{r}$ and $\omega_{d}$ of rates $\mathrm{R}_{r}$ and $\mathrm{R}_{d}$, respectively, where

$$
\mathrm{R}=\mathrm{R}_{r}+\mathrm{R}_{d}
$$

The first packet is transmitted to the relay during slot 1 , which can decode it if:

$$
\mathrm{R}_{r} \leq \alpha \cdot \log _{2}\left(1+\left|h_{s, r}\right|^{2} \frac{\mathrm{P}_{s}}{N_{o}}\right)
$$

The packet also reaches the destination. During the second time slot, the source transmits the packet $\omega_{d}$ directly to destination with a fraction $1-\beta \in[0,1]$ of the total available power for that time slot. Simultaneously, the source and the relay retransmit the first packet $\omega_{r}$ using the opportunistic approach defined in Subsection IV-A. To do so, they use the remaining fraction of power for slot $\beta$. Hence, following arguments in [8], the destination is able to decode $\omega_{r}$ if:

$$
\begin{aligned}
\mathrm{R}_{r} & \leq \alpha \cdot \log _{2}\left(1+\left|h_{s, d}\right|^{2} \frac{\mathrm{P}_{s}}{N_{o}}\right) \\
& +(1-\alpha) \log _{2}\left(1+\frac{\beta \max \left\{\left|h_{s, d}\right|^{2} \mathrm{P}_{s},\left|h_{r, d}\right|^{2} \mathrm{P}_{r}\right\}}{N_{o}+\left|h_{s, d}\right|^{2}(1-\beta) \mathrm{P}_{s}}\right)
\end{aligned}
$$

Notice that the power utilized to transmit $\omega_{d}$ acts as noise when decoding $\omega_{r}$. Moreover, the destination decodes $\omega_{d}$

$$
\mathrm{R}_{d} \leq(1-\alpha) \cdot \log _{2}\left(1+\left|h_{s, d}\right|^{2} \frac{(1-\beta) \mathrm{P}_{s}}{N_{o}}\right)
$$

Finally, adding (13) and (15) we have the maximum transmission rate with relay, while adding (14) and (15) we have the maximum rate for decoding at the destination (See [8] for an entire proof). Thus, the maximum transmission rate is the minimum of both as in (11), at the top of this page. As in opportunistic relaying, the total power transmitted by the source and relay must satisfy the CS region constraint in (10).

\section{NUMERICAL RESUlts}

The performance of the proposed cooperative strategy has been evaluated numerically. A wireless backhaul with a linear topology and up to 10 wireless virtual links has been considered (each virtual link consists of the two end nodes plus an intermediate node). All links are assumed to have equal transmit power and to be separated by the same distance, which results in equal average SNR. A path loss exponent of 3 has been assumed. Two propagation models have been simulated: Rayleigh fading, representing connectivity in nonline of sight condition; and Rice fading with $k=6 \mathrm{~dB}$, representing connectivity in line of sight condition. The Rice model is more appropriate for a rooftop interconnection, or for a system using directional antennas.

Figure 4 shows the end-to-end throughput of the cooperative wireless backhaul as a function of the number of links, for
Rayleigh fading. The average SNR between consecutive links is $10 \mathrm{~dB}$. Results under both infinite buffer and no-buffer assumptions are shown. As it can be seen in the figure, in both cases the throughput improves with the use of cooperative techniques, although the improvement is larger in the no-buffer case. The difference between opportunistic relaying and partial decoding is, however, rather small. Similar conclusions can be drawn from the results in Figure 5 for the Rice fading case. The throughput obtained is superior to the Rayleigh channel throughput with all methods, but the relative advantage of cooperative strategies is somewhat smaller.

Figure 6 shows the end-to-end outage probability of the cooperative wireless backhaul, also as a function of the number of links. The rates of $1 \mathrm{bit} / \mathrm{s} / \mathrm{Hz}$ and $2 \mathrm{bit} / \mathrm{s} / \mathrm{Hz}$ are considered. From the figure, we can see that the virtual linkbased backhaul is more robust to channel fluctuations, with an improvement in outage probability in some cases close to an order of magnitude. Results for the Rice channel are shown in Figure 7, where all methods show higher robustness, and the cooperative backhaul also outperforms the non-cooperative one.

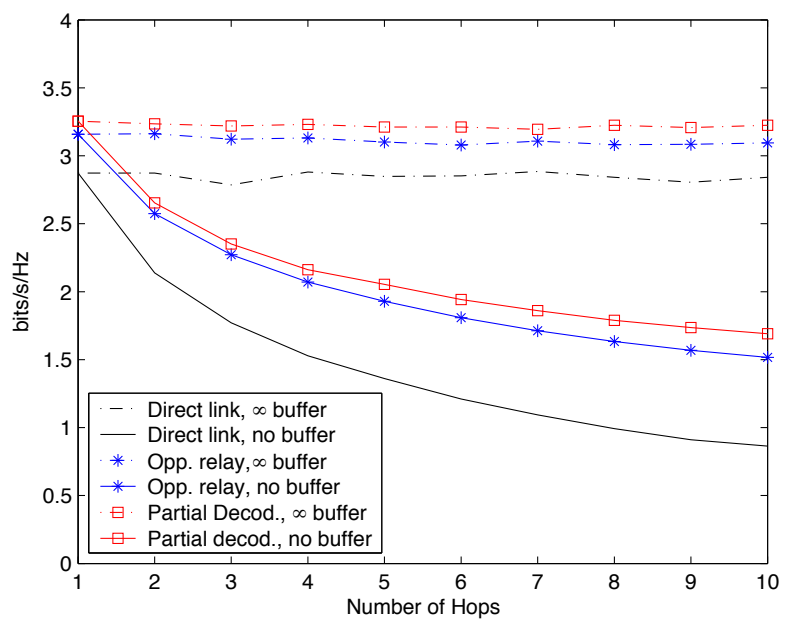

Fig. 4. End-to-end throughput as a function of the number of links. Rayleigh fading. Average SNR $=10 \mathrm{~dB}$

\section{CONCLUSIONS}

A cooperative wireless backhaul was proposed for a wireless mesh network. In the proposed system, cooperative techniques are used to establish virtual links among nodes in the wireless backhaul. The purpose of virtual links is to hide cooperative strategies from the network layer, so that traditional (noncooperative) routing protocols can be used. Within a virtual link, which is defined by the carrier sensing and connectivity regions, nodes cooperate using opportunistic relaying or partial decoding. The end-to-end performance of the cooperative backhaul was evaluated in terms of throughput and outage probability. A wireless backhaul with a linear topology was evaluated, both for line of sight and non-line of sight propagation. Simulation results show remarkable gains, in both 


$$
\begin{aligned}
\mathrm{R}=\max _{\alpha, \beta} \min \left\{\alpha \cdot \log _{2}\left(1+\left|h_{s, r}\right|^{2} \frac{\mathrm{P}_{s}}{N_{o}}\right)+(1-\alpha) \cdot \log _{2}\left(1+\left|h_{s, d}\right|^{2} \frac{(1-\beta) \mathrm{P}_{s}}{N_{o}}\right),\right. \\
\left.\alpha \cdot \log _{2}\left(1+\left|h_{s, d}\right|^{2} \frac{\mathrm{P}_{s}}{N_{o}}\right)+(1-\alpha) \log _{2}\left(1+\frac{\left|h_{s, d}\right|^{2}(1-\beta) \mathrm{P}_{s}+\beta \max \left\{\left|h_{s, d}\right|^{2} \mathrm{P}_{s},\left|h_{r, d}\right|^{2} \mathrm{P}_{r}\right\}}{N_{o}}\right)\right\}
\end{aligned}
$$

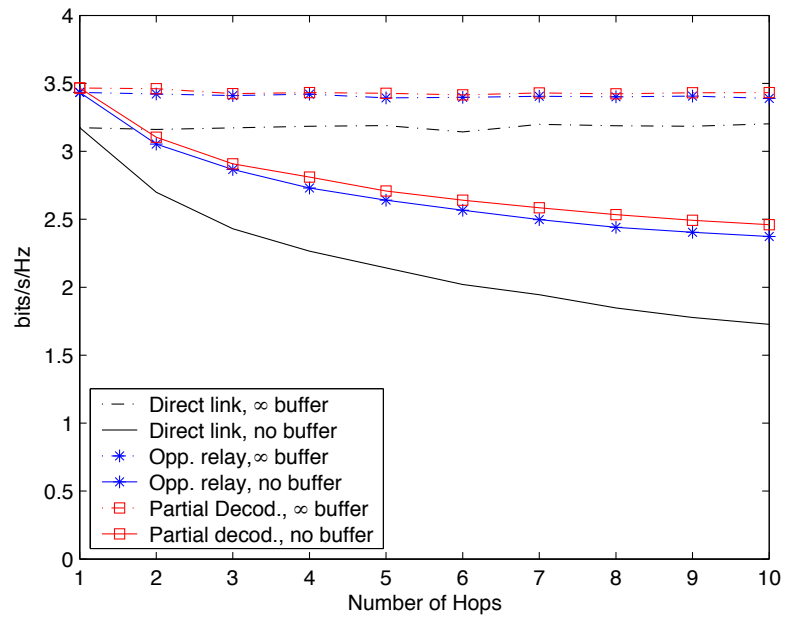

Fig. 5. End-to-end throughput as a function of the number of links. Rice fading. Average SNR $=10 \mathrm{~dB}$.

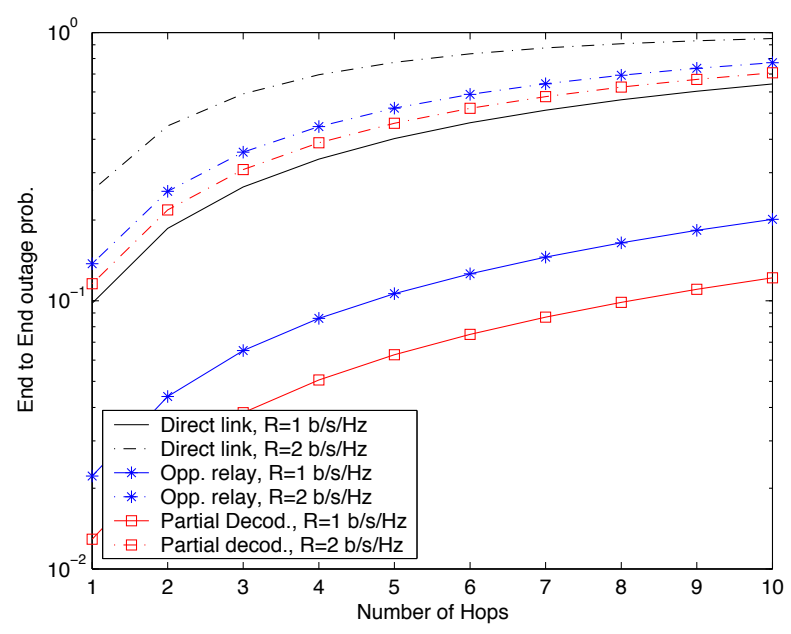

Fig. 6. Outage probability of the end-to-end link for spectral efficiencies of 1 and $2 \mathrm{~b} / \mathrm{s} / \mathrm{Hz}$. Rayleigh fading. Average SNR $=10 \mathrm{~dB}$.

metrics, when cooperative techniques are used.

\section{ACKNOWLEDGEMENT}

This work has been performed within the framework of IST project 27402, WIP, which is partly funded by the European
Union.

\section{REFERENCES}

[1] "http://seattlewireless.net/,"

[2] R. Ramanathan, "Challenges: a radically new architecture for next generation mobile ad hoc networks," in Proceedings of the 11th annual international conference on Mobile computing and networking, 2005, pp. $132-139$.

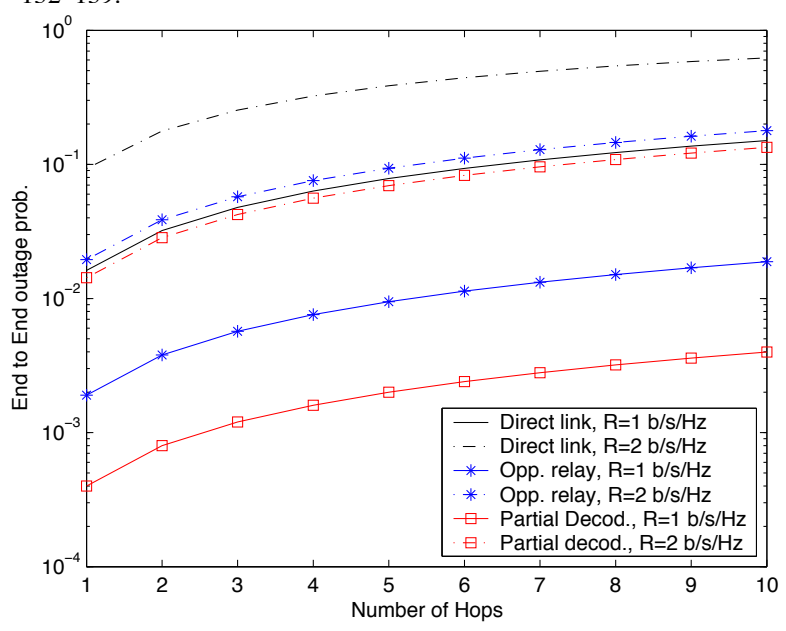

Fig. 7. Outage probability of the end-to-end link for spectral efficiencies of 1 and $2 \mathrm{~b} / \mathrm{s} / \mathrm{Hz}$. Rice fading. Average SNR $=10 \mathrm{~dB}$.

[3] R. Karrer, A. Sabharwal, and E. Knightly, "Enabling large-scale wireless broadband: The case for taps," ACM SIGCOMM Computer Communication Review, vol. 34, no. 1, pp. 27-31, Jan. 2004.

[4] P. Liu, Z. Tao, Z. Lin, E. Erkip, and S. Panwar, "Cooperative wireless communications: a cross-layer approach," IEEE Wireless Communications, pp. 84-92, Aug. 2006.

[5] T. Cover and A. El Gamal, "Capacity theorems for the relay channel," IEEE Transactions on Information Theory, vol. 25, no. 5, pp. 572-584, Sept. 1979.

[6] J. N. Laneman and G. Wornell, "Distributed space-time coded protocols for exploiting cooperative diversity in wireless networks," IEEE Transactions on Information Theory, vol. 49, no. 10, pp. 2415-2425, Oct. 2003.

[7] A. Sendonaris, E. Erkip, and B. Aazhang, "User cooperation diversity (parts i and ii)," IEEE Transactions on Communications, vol. 51, no. 11, pp. 1927-1948, Nov. 2003.

[8] A. del Coso and C. Ibars, "Partial decoding for synchronous and asynchronous Gaussian multiple relay channels," in Proc. International Conference on Communications (ICC), Glasgow, UK, Jun. 2007, to appear. 